\title{
Novel Methods to Address Antimicrobial Resistance
}

\author{
Tim Sandle* \\ Head of Microbiology at Bio Products Laboratory, UK
}

Received: February 05, 2014; Accepted: February 14, 2014; Published: February 17, 2014

*Corresponding author: Tim Sandle, Bio Products Laboratory, 68 Alexandra Road, London Colney, St. Albans Hertfordshire, UK, Tel: 0-780-890-6409;

E-mail: timsandle@btinternet.com

\section{Introduction}

Antimicrobial resistance describes the ability of a microorganism to resist the action of antimicrobial drugs. In a few instances some microorganisms are naturally resistant to particular antimicrobial agents; however, a more common problem is when microorganisms that are normally susceptible to the action of particular antimicrobial agents develop resistance. Such resistance invariably arises as a result of changes in the microbial genes (a spontaneous or induced genetic mutation). In some cases, the genes causing resistance can be transferred between different strains of microorganism (horizontal gene transfer via conjugation, transduction, or transformation). When the latter happens the recipient organisms will also become resistant.

Many antibiotic resistance genes reside on transmissible plasmids (a small DNA molecule that is able to replicate independently of the chromosomal DNA contained within a cell), facilitating their transfer [1]. Exposure to an antibiotic naturally selects for the survival of the microorganisms that possess the genes for resistance. Based on this, a gene for antibiotic resistance can potentially spread through a bacterial ecosystem. The phenomenon of antimicrobial resistance has been accelerating over the past three decades. The causes of antimicrobial resistance include the over-prescribing by doctors, often for conditions which do not require antibiotics [2]; and the addition of antibiotics to animal feeds [3].

Humans face the very real risk of a future without antibiotics. The implications of this are that life expectancy could fall due to people dying from diseases that are readily treatable today. One concern for the future is the re- emergence of diseases that are 'officially' extinct, such as tuberculosis (a disease caused by Mycobacterium tuberculosis). The other concern is that without effective antibiotics, human society will no longer be able to conduct the types of medical procedures that can lead to immunosuppression. Such therapies include those for cancer treatments or to address autoimmune disorders.

In terms of microbial risks, Penicillin- resistant pneumococci were widespread internationally and vancomycin- resistant enterococci were also circulating in hospital specialist units. Methicillin- resistant Staphylococcus aureus (MRSA) were relatively uncommon in serious infections at the start of the 1990s; however, these have since proliferated since the early 2000s. To add to this catalogue, in 2013 it was reported that gonorrhoea is becoming resistant to most antimicrobials. Furthermore, many routine surgeries may also become too dangerous to perform owing to the risk of untreatable infection.

Whilst many governments are placing restrictions on antibiotic use [4], this is too little, too late. The current situation has made the quest for new antibiotics and antibiotic alternatives a matter of great importance. Given the development costs, the complexity in finding new drug targets, and the timescales involved, the prospect of antibiotic alternatives remains low; therefore, greater prospects lie with alternative approaches. This editorial considers some of the current initiatives which form part of the quest for antimicrobial alternatives.

There are some signals that alternative antimicrobial drugs could be emerging. This is a slow process requiring the testing of new molecules as potential antibiotics. One main centre of analysis is the laboratory of Kenneth Keiler (from Penn State University, USA). The research team here has examined 663,000 different molecules against a strain of Escherichia coli bacteria. The researchers have monitored how the chemicals affect the growth and survival of the bacterium. From this, forty-six potential chemicals have been selected. Each of the selected molecules targets the protein synthesis in bacteria and disrupts the process. This results in the bacterium being unable to replicate. By halting bacterial growth, infection is unable to spread [5].

An alternative approach is to be found in a paper by Geller $\mathrm{BL}$, et al. [6], a new bacterial-killing chemical is described [6]. The new antibacterial agent is termed as Peptide-conjugated phosphorodiamidate morpholino oligomers (PPMO). The chemical is a synthetic analogue of DNA or RNA that has the ability to silence the expression of specific genes within bacterial cells. The PPMOs being developed are chemically synthesized. In animal studies, one form of PPMO showed significant control of two strains of the bacteria Acinetobacter, a group of bacteria of global medical concern.

One concern with antimicrobials is that they destroy beneficial bacteria as well are harmful ones. To overcome this, a research team is exploring ways to target specific bacteria by focusing on the CRISPR-Cas system, which forms part of the immune system 
in many bacteria (functioning to protect bacteria from viruses). The model generates strands of RNA termed CRISPR RNAs. These molecules match DNA sequences that are specific to certain viruses. Once CRISPR RNAs locate a specific bacterium, they release Cas proteins that slice through the DNA. North Carolina State University are examining how to turn CRISPR RNAs to target DNA sequences in the bacteria themselves, causing bacterial cell death. The main aim is to do so in a way that only eliminates a targeted strain [7].

Taking another approach, some scientists argue that adding silver to existing antibiotics can increase their effectiveness Writing in Science Translational Medicine in 2013, researchers have explained the cellular processes by which the precious metal weakens bacteria and makes them more susceptible to antibiotics [8].

A different consideration is with developing a candidate drug that does not destroy pathogenic bacteria, but rather disarms them. Researchers based at VIB/Vrije Universiteit Brussel are exploring ways to inhibit the pilus formation mechanism (a means of bacterial attachment) [9].

\section{Conclusion}

Whilst the rise of antibiotic bacteria remains a matter of great seriousness and the quest for new antibiotics remains unfulfilled, some of the research initiates highlighted present promising candidates. It is important, in the next few years that a sustainable supply of new, effective antimicrobials emerges. Alongside this rapid diagnostics and complementary tools for use in health, social care, and veterinary systems are required. There are few public health issues of greater importance than antimicrobial resistance in terms of impact on society.

\section{References}

1. Lipps G (2008) Plasmids: current research and future trends. Caister Academic Press, UK.

2. CDC (2013) Antibiotic resistance threats in the United States, 2013. Centers for Disease Control and Prevention, CDC, Washington, USA.

3. Martinez JL, Olivares J (2012) Environmental pollution by antibiotic resistance genes. In PL Keen, MH Montforts (eds.) Antimicrobial resistance in the environment. John Wiley \& Sons, Hoboken, New Jersey, pp: 151- 171.

4. Barnett ML, Linder JA (2014) Antibiotic prescribing to adults with sore throat in the United States, 1997-2010. JAMA Intern Med 174(1): 138-140.

5. Ramadossa NS, Alumasaa JN, Lin C, Yu W, Li S, et al (2013) Small molecule inhibitors of trans-translation have broad-spectrum antibiotic activity. PNAS.

6. Geller BL, Marshall-Batty K, Schnell FJ, McKnight MM, Iversen PL, et al. (2013) Gene-silencing antisense oligomers inhibit acinetobacter growth in vitro and in vivo. J Infect Dis 208(10): 1553-1560.

7. Gomaa AA, Klumpe HE, Luo ML, Kurt S, Barrangoub R, et al. (2014) Programmable removal of bacterial strains by use of genometargeting CRISPR-Cas Systems. mBio 5 (1): 13.

8. Morones- Ramirez JR, Winkler JA, Spina CS, Collins JJ (2013) Silver enhances antibiotic activity against gram- negative bacteria. Sci Transl Med 5 (190): 190ra81.

9. Alvin W H Lo, Karen V de Water, Paul J Gane, AW Edith Chan, Steadman D, et al. (2013) Suppression of type 1 pilus assembly in uropathogenic Escherichia coli by chemical inhibition of subunit polymerization. J Antimicrob Chemother. 